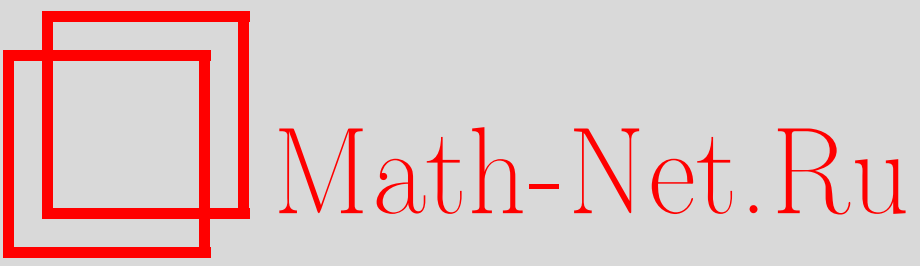

Заседания межвузовского научного семинара «Прикладная математика и механика» при Самарском государственном техническом университете (сентябрьдекабрь, 2009 г.), Вестн. Сам. гос. техн. ун-та. Сер. Физ.-мат. науки, 2010, выпуск 1(), 263-274

DOI: https://doi.org/10.14498/vsgtu755

Использование Общероссийского математического портала Math-Net.Ru подразумевает, что вы прочитали и согласны с пользовательским соглашением

http://www.mathnet.ru/rus/agreement

Параметры загрузки:

IP : 3.89 .197 .203

26 апреля 2023 г., 09:14:52 


\section{Заседания межвузовского научного семинара «Прикладная математика и механика» \\ при Самарском государственном техническом университете (сентябрь-декабрь, 2009 г.)}

Ниже приводятся подготовленные авторами резюме докладов, обсуждавшихся на семинаре.

10.09.2009. В. E. Зотеев (докторант) каф. прикладной математики и информатики Самарского государственного технического университета

«Линейно-параметрические дискретные модели в форме разностных уравнений в задачах идентификации диссипативных механических систем» (доклад по материалам диссертации на соискание учёной степени доктора наук по специальности 05.13.18)

Важнейшей проблемой в машиностроении является проблема идентификации нелинейных диссипативных механических систем в процессе их эксплуатации или прочностных промышленных испытаний. Это объясняется тем, что основным диагностическим признаком технического состояния диссипативной механической системы являются ее динамические характеристики, в том числе показатель нелинейности системы. Результаты многочисленных исследований на конкретных примерах подтверждают непосредственную связь между техническим состоянием различного рода механических систем (например, усталостным разрушением материалов, возникновением и развитием микротрещин в деталях, появлением недопустимых люфтов в узлах конструкций, значительным износом контактирующих поверхностей, технологическим браком при сборке и т.п.) и её динамическими характеристиками.

Решить задачу повышения достоверности и оперативности определения динамических характеристик диссипативной системы можно только на основе новых математических моделей, описывающих результаты наблюдений динамического процесса на выходе системы и ориентированных на современный уровень компьютеризации исследований и применение статистических методов обработки экспериментальных данных.

В настоящее время существуют различные подходы и способы определения динамических характеристик механической колебательной системы. Среди них лидирующее место занимают высокоэффективные методы вибродиагностики, ориентированные на применение современных средств и алгоритмов вычислений и обработки информации, например, методы цифрового спектрального анализа, методы корреляционного анализа. Основу этих методов составляют стохастические параметрические модели временных рядов.

Однако область применения этих методов функционально ограничена и исключает задачи, в которых основным диагностическим признаком технического состояния механической системы является характеристика рассеяния колебательной энергии, в том числе характеристика нелинейности диссипативной силы. Такие задачи возникают, в частности, при разработке гидравлических амортизаторов, исследованиях конструкционного демпфирования, то есть демпфирования, обусловленного потерями на трение в неподвижных соединениях (прессовых, заклепочных, резьбовых, шлицевых и т.п.), или внутреннего трения в материале при его циклическом деформировании.

Широко применяемые на практике методы определения характеристик рассеяния энергии колебаний различных механических конструкций и демпфирующих свойств материалов совершенно не вписываются в формат современных информационных технологий, применяемых в вибродиагностике. Как правило, эти методы 
громоздки, нередко требуют графических построений, применяемые алгоритмы вычислений построены на линеаризованных детерминированных моделях и используют минимально необходимое число точек эксперимента при полном отсутствии процедур, связанных со статистической обработкой результатов наблюдений.

Таким образом, необходимость коренного улучшения качества машиностроительных конструкций требует разработки и применения при диагностике технического состояния большого класса МС новых высокоточных, оперативных методов определения динамических характеристик, в том числе характеристик нелинейности механической системы как диагностического признака ее технического состояния, методов, соответствующих современному уровню компьютеризации и автоматизации исследований динамических процессов в машинах и механизмах. Основой разработки таких методов могут стать стохастические параметрические модели временных рядов, описывающие результаты наблюдений мгновенных значений динамического процесса на выходе системы при типовых тестовых воздействиях.

Объектом исследования являются нелинейные диссипативные механические системы с одной и несколькими степенями свободы, а также с распределенными параметрами, к которым относятся, например, конструкционные материалы. Рассматриваются системы с диссипативными силами, пропорциональными $n$-степени скорости движения, в том числе системы с линейно-вязким, турбулентным и кулоновым трением, а также системы с гистерезисным трением.

Предметом исследования являются математические модели, описывающие в форме стохастических разностных уравнений результаты измерений мгновенных значений динамического процесса на выходе диссипативной механической системы, а также численный метод определения динамических характеристик диссипативной механической системы на основе среднеквадратичного оценивания коэффициентов разностных уравнений.

Целью работы является разработка нового научного подхода к решению проблемы идентификации диссипативных механических систем, в основе которого лежат линейно-параметрические дискретные модели, описывающие в форме разностных уравнений результаты наблюдений динамического процесса на выходе системы. Для достижения поставленной цели автором были поставлены и решены следующие взаимосвязанные научные задачи:

- разработка теоретических основ и принципов построения линейно-параметрических дискретных моделей, описывающих динамические процессы в диссипативных системах;

- формирование класса и систематизация линейно-параметрических дискретных моделей, описывающих в форме стохастических разностных уравнений результаты измерений мгновенных значений колебаний диссипативной механической системы;

- разработка численного метода определения динамических характеристик диссипативных систем на основе среднеквадратического оценивания коэффициентов стохастического разностного уравнения, позволяющего обеспечить высокую помехозащищенность оценок за счет эффективного использования статистических методов обработки экспериментальных данных;

- анализ и оценка погрешности результатов вычисления динамических характеристик диссипативных систем на основе стохастических разностных уравнений, в том числе исследование устойчивости вычисления среднеквадратичных оценок коэффициентов разностного уравнения; разработка и исследование эффективности структурных методов повышения устойчивости вычисления среднеквадратичных оценок коэффициентов разностного уравнения;

- разработка программного обеспечения, реализующего устойчивые алгоритмы вычислений динамических характеристик и предназначенного для использования в физических экспериментах. 
В работе получены следующие новые научные результаты:

- разработан новый научный подход к решению задачи определения динамических характеристик нелинейной диссипативной механической системы;

- разработаны основы теории и техники построения линейно-параметрических моделей, связывающих дискретные значения функциональных зависимостей, описывающих динамические процессы в диссипативных системах;

- построены и систематизированы в зависимости от типа нелинейности системы и вида тестового воздействия линейно-параметрические дискретные модели, отличающиеся от известных своей структурой и тем, что коэффициенты этих моделей известным образом связаны с динамическими характеристиками системы;

- разработан численный метод определения динамических характеристик диссипативной системы на основе линейно-параметрических дискретных моделей, новизна которого заключается в том, что задача вычисления параметров диссипативной системы сводится к среднеквадратичному оцениванию коэффициентов разностного уравнения;

- разработаны структурные методы повышения устойчивости вычисления среднеквадратичных оценок, в основе которых лежат модифицированные линейнопараметрические дискретные модели, отличающиеся наличием параметра, который позволяет обеспечить высокую устойчивость вычислений;

- построены новые линейно-параметрические дискретные модели, описывающие результаты эксперимента при неупругом реологическом деформировании материалов и элементов конструкций, и на их основе разработаны высокоточные алгоритмы определения параметров кривой ползучести для нового класса задач оценки индивидуальной надежности механических систем;

- построены новые линейно-параметрические дискретные модели, описывающие в форме разностных уравнений результаты измерений огибающей амплитуд колебаний систем с диссипативными силами, пропорциональными $n$ степени скорости движения, в том числе систем с кулоновым, линейно-вязким и турбулентным трением, лежащие в основе новых численных методов вычисления диссипативных характеристик системы по огибающей амплитуд колебаний; разработаны новые специализированные устройства для измерения различных диссипативных характеристик (декремента колебаний, показателя затухания) в системах с линейно-вязким, турбулентным и кулоновым трением, отличающиеся от аналогов более высокой точностью.

Применение разработанных методов определения параметров механической системы на основе разностных уравнений обеспечивает существенное повышение точности вычисления диссипативных характеристик, а, следовательно, и достоверности оценки технического состояния механической системы. При этом по сравнению с известными методами определения параметров диссипативной системы по огибающей амплитуд колебаний или по резонансной кривой точность оценивания повышается в среднем на порядок. Применение итерационной процедуры среднеквадратичного оценивания коэффициентов разностного уравнения позволяет за счет устранения смещения уменьшить погрешность оценок по сравнению с известными алгоритмами вычислений в несколько раз. Построенные модели позволяют с высокой точностью оценить показатель нелинейности механической системы, который является важнейшим диагностическим признаком ее технического состояния, что существенно расширяет функциональные возможности разработанного метода определения параметров диссипативной системы. Применение линейно-параметрических дискретных моделей обеспечивает высокую оперативность получения оценок диссипативных характеристик (за несколько периодов колебаний), что позволяет использовать 
разработанные алгоритмы в задачах управления в режиме реального времени (online). Таким образом, научные результаты, представленные в работе, позволяют коренным образом изменить способы вычисления диссипативных характеристик при оценке технического состояния механической системы за счет внедрения в практику вибродиагностики диссипативных систем современных компьютерных технологий и статистических методов обработки экспериментальных данных

17.09.2009. C. В. Воронин (аспирант) каф. технологии металлов и авиационного материаловедения Самарского государственного аэрокосмического университета им. ак. С. П. Королёва

«Компьютерное моделирование реальной структуры металлических материалов при исследовании процессов деформации и разрушения» (доклад по материалам диссертации на соискание учёной степени кандидата наук по специальностям 05.02.01, 01.02.04)

Современные отрасли машиностроения постоянно нуждаются в полуфабрикатах и деталях, к которым предъявляются высокие требования по качеству, связанные с физико-механическими свойствами, геометрической точностью изготовления, шероховатостью поверхности и рядом других параметров. Все приведенные свойства, как известно, в определенной степени зависят от структуры материалов. Поэтому при исследовании процессов деформации и разрушения необходимо более адекватно учитывать структуру материала, а в частности форму, размер и свойства структурных составляющих.

В настоящее время широкомасштабно используются компьютерные системы инженерного моделирования, анализа и оптимизации - CAE (Computer Aided Engineering), такие как MSC.Nastran, MSC.Marc, MSC.SuperForm. Перечисленные программные продукты позволяют решать соответствующие краевые задачи механики деформируемого твердого тела и моделировать с применением метода конечных элементов (MКЭ) технологические процессы деформации и разрушения, поведение различных систем, элементов конструкций, механизмов при внешних воздействиях, тем самым, сокращая объем дорогостоящих лабораторных и производственных экспериментов. Однако большинство моделей технологических процессов, создаваемых в вышеперечисленных программах, рассматривают обрабатываемый материал изотропным, без учета его структурных составляющих и анизотропии, в итоге снижается точность определения напряженно-деформированного состояния (НДС) обрабатываемой заготовки и инструмента, а также основных технологических параметров процессов.

Следовательно, разработка методов решения краевых задач и компьютерного моделирования реальной структуры металлических материалов при исследовании процессов деформации и разрушения на основе современного программного и математического обеспечения для инженерного анализа, является актуальным научным направлением.

Для повышения эффективности и точности математического моделирования путем разработки и апробации методических подходов к решению краевых задач, компьютерному исследованию и визуализации процессов деформации, разрушения с учетом структуры металлических материалов решались следующие конкретные задачи:

- разработка методики создания 2D и $3 \mathrm{D}$ конечно-элементных моделей объектов исследования с учетом реальной структуры материала;

- проведение моделирования процесса растяжения плоских образцов из сплава АМг6 и АД1 с учетом реальной структуры и выполнить проверку адекватности реальным экспериментальным исследованием;

- установление целесообразности учёта реальной структуры материала при моделировании процессов прокатки плоских образцов и осадки цилиндрического образца из сплава АМг6 
- решение ряда краевых задач методом конечных элементов и разработанными методиками, проведение компьютерного исследования процесса фестонообразования при вытяжке полой цилиндрической детали из сплава АМг6 и АД1М;

- решение краевой задачи, визуализация и исследование закономерностей процесса распространения трещины в плоском образце из анизотропного хрупкого материала методом компьютерного моделирования.

Впервые разработаны методики создания 2D и 3D конечно-элементных моделей (KЭМ) объектов исследования с учетом реальной структуры материала, на примере алюминиевых сплавов АМг6 и АД1М. Разработаны программы-приложения автоматизированного построения моделей с учетом реальной структуры и кристаллографической ориентации зерен различных фаз для пакета программ MSC.Nastran for Windows.

24.09.2009. B. В. Абрамов (аспирант) каф. прикладной математики и информатики Самарского государственного технического университета

«Программное обеспечение и математическое моделирование движения малых тел Солнечной системы на основе методов Адамса» (доклад по материалам диссертации на соискание учёной степени кандидата наук по специальности 05.13.18)

В последние годы достижения в области математического моделирования и вычислительного эксперимента как информационной технологии получения новых знаний об окружающем нас мире приобретают все большее значение для различных областей наук.

В связи с увеличением объема информации о динамических параметрах малых тел Солнечной системы возрос интерес к проблеме «астероидной опасности». В настоящее время известно свыше шести тысяч астероидов, проникающих внутрь орбиты Марса и Земли. Наибольшую опасность для Земли, наряду с кометами, представляют астероиды групп Аполлона, Амура, Атона.

Теория движения астероидов групп Аполлона, Амура, Атона значительно сложнее теории движения планет, поскольку эллиптические орбиты астероидов более вытянуты, чем орбиты планет, плоскости орбит значительно наклонены к плоскости эклиптики. Кроме того, астероиды имеют тесные сближения с большими планетами. Поскольку высокоэффективные аналитические теории движения астероидов, имеющих сближения, не разработаны, для исследования эволюции их орбит широко применяются численные методы.

Исследование их эволюционных процессов, устойчивости движения, оценка вероятности столкновения и предотвращение катастрофических последствий является лишь неполным перечнем проблем, требующих решения. Разработка численных методов для решения уравнений движения небесных тел является одним из составных этапов при решении проблемы, связанной с «астероидной опасностью».

Целью работы является разработка вычислительных алгоритмов многошаговых методов Адамса с высоким порядком аппроксимирующих формул и программного обеспечения на их основе для исследования движения небесных тел.

В соответствии с указанной целью необходимо было решение следующих задач:

- разработка вычислительных алгоритмов методов Адамса с высоким порядком аппроксимации для решения обыкновенных дифференциальных уравнений;

- создание математического и программного обеспечения для исследования движения астероидов групп Аполлона, Амура, Атона, сближающихся с Землей;

- создание информационной базы данных малых тел Солнечной системы (астероидов групп Аполлона, Амура, Атона и короткопериодических комет) на интервале времени 400 лет (1800-2206 гг.);

- выявление наиболее опасных объектов и классификация астероидов, представляющих потенциальную угрозу для Земли в случае столкновения с ней; 
- создание Интернет-ресурса и размещение на нем полной информации об эволюции орбит малых тел Солнечной системы.

Разработанные в работе вычислительные алгоритмы универсальных многошаговых методов Адамса, в отличие от ранее существующих алгоритмов, обладают высоким (до 16-го включительно) порядком аппроксимирующих формул и могут применяться для решения различного рода задач описываемых дифференциальными уравнениями.

Информационная база данных орбитальной эволюции малых тел Солнечной системы, размещенная на Интернет-ресурсе SmallBodies. Ru может быть полезной как для учебной, так и научных целей.

22.10.2009. E. В. Биткина (аспирант) каф. механики Самарского государственного технического университета

«Разработка метода анализа напряжённо-деформированного состояния многослойных композиционных материалов и конструкций с учетом температурных, силовых и технологических воздействий» (доклад по материалам диссертации на соискание учёной степени кандидата наук по специальности 01.02.04)

Композиционные материалы находят все более широкое применение в различных отраслях техники, что объясняется широким спектром свойств, выгодно отличающих их от традиционных материалов и сплавов: высокая удельная прочность, жаростойкость, усталостная и длительная прочность и т. д. Данный факт объясняет необходимость исследований, направленных на создание новых конструкций из композитов. Методам механики деформируемого твёрдого тела в совокупности этих исследований принадлежит одно из главных мест. Действительно, три основных проблемы - механика собственно композита (определение физико-механических свойств и задачи синтеза); методы расчета конструкций и их элементов на прочность, устойчивость, долговечность и задачи оптимизации; технология создания композиционных материалов и конструкций из них - решаются преимущественно методами механики. Аналогичные задачи возникают и при создании инженерных сооружений из традиционных материалов, однако удельный вес и значимость решения задач механики деформирования в каждой из перечисленных проблем применительно к композитам существенно выше.

Работы по созданию методов исследования напряжённо-деформированного состояния композиционных материалов и внедрению этих материалов в различные конструкции ведутся в настоящее время достаточно широко. Однако, вопросам анализа влияния анизотропии термомеханических свойств, остаточных температурных напряжений и деформаций неоднородных слоистых конструкций, предварительного натяжения армирующих волокон, асимметрии свойств структуры пакета композиционного материала по толщине и ряда других факторов на напряжённо-деформированное состояние композита не уделено должного внимания. В качестве примера отметим, что в реальных конструкциях из композиционных материалов «случайна» анизотропия материала, связанная с технологическими погрешностями и дефектами чередующихся слоёв, проявляется в условиях эксплуатации как отклонение от расчётной теоретической формы.

Таким образом, задача теоретических исследований заключается не только и не столько в том, чтобы определить механические свойства данного композиционного материала, а в том, чтобы на основе этих исследований сконструировать композит с наперед заданными деформационными и прочностными характеристиками. Сформулированная в таком виде задача является достаточно сложной и требует проведения дополнительных исследований и разработок в этой области.

Разработка метода анализа напряжённо-деформированного состояния многослойных композиционных материалов и конструкций с учетом температурных, силовых и конструктивно-технологических факторов - угла разориентации слоёв композиционных материалов, натяжения слоев препрега, степени армирования. Дости- 
жение этой цели требует комплексного подхода, поскольку получение наивысших эксплуатационных характеристик конструкций, изготовленных из композиционных материалов, находится в прямой зависимости от технологии, конструктивных параметров и условий внешнего нагружения. В этой связи в работе решаются следующие основные задачи:

- разработка математической модели процесса деформирования многослойных композиционных материалов и изготовленных из них конструкций, параметрический анализ конструктивно-технологических факторов;

- анализ и синтез структуры композиционного материала конструкции с учётом технологических воздействий по критериям жесткости, прочности, размерной стабильности;

- проведение экспериментальных исследований по определению величин механических характеристик и сравнение полученных результатов с теоретическими для проверки адекватности разработанного метода;

- разработка рекомендаций по созданию формостабильных композитных конструкций.

Разработан метод анализа НДС многослойных композиционных материалов и конструкций на основе математической модели, позволяющей решать связанную задачу, не разделяя ее на плоскую и изгиб. Система дифференциальных уравнений сведена к одному уравнению восьмого порядка. Задача решается аналитически в рамках классической теории упругости пластин и оболочек.

03.11.2009. E. B. Стебанюћ (докторант) каф. прикладной математики и информатики Самарского государственного технического университета

«Модельные представления аналитических решений краевых задач теории теплообмена на основе введения дополнительных граничных условия» (доклад по материалам диссертации на соискание учёной степени доктора наук по специальности 05.13.18)

Известно, что точные аналитические решения краевых задач математической физики в настоящее время получены лишь для отдельных частных случаев и, к тому же, при весьма существенных допущениях (неучёт нелинейности, переменности физических свойств и граничных условий и проч.). Кроме того, решения, полученные с помощью классических аналитических методов, представляются в форме бесконечных рядов, плохо сходящихся в окрестностях граничных точек и при малых значениях временно́й координаты. Исследования показывают, что сходимость точного аналитического решения нестационарной задачи теплопроводности для бесконечной пластины при граничных условиях первого рода в диапазоне чисел Фурье $10^{-12} \leqslant \mathrm{Fo} \leqslant 10^{-7}$ на- блюдается лишь при использовании от 1000 (Fo $\left.=10^{-7}\right)$ до пятисот тысяч $\left(\mathrm{Fo}=10^{-12}\right)$ членов ряда. В тоже время, исследование кинетики теплового процесса при временах микросекундной длительности исключительно важная в практическом отношении проблема.

В аналитической теории теплопроводности известны методы, в которых используется понятие глубины термического слоя (интегральные методы теплового баланса). K ним, в частности, относятся интегральный метод теплового баланса, метод осреднения функциональных поправок, методы М. Е. Швеца, М. Био, А. И. Вейника и др.

Несомненным их преимуществом является возможность получения простых по форме аналитических решений удовлетворительной точности как для регулярного, так и нерегулярного процессов теплопроводности. Однако их серьезным недостатком является низкая точность. Причина в том, что получаемое решение, точно удовлетворяя начальному и граничным условиям, основному дифференциальному урав- 
нению удовлетворяет лишь в среднем. Это связано с тем, что в основу интегральных методов положено построение так называемого интеграла теплового баланса, что равнозначно осреднению исходного дифференциального уравнения в пределах глубины термического слоя. Следовательно, очевидным путем повышения точности интегральных методов является улучшение выполнения исходного дифференциального уравнения. С этой целью в настоящей работе избрано направление аппроксимационного представления приближенного решения с определением любого числа его слагаемых. Для определения неизвестных коэффициентов таких полиномов основных граничных условий оказывается недостаточно. В связи с чем, возникает необходимость привлечения дополнительных граничных условий, определяемых из исходного дифференциального уравнения с использованием основных граничных условий и условий, задаваемых на фронте температурного возмущения.

В работе поставлены и решаются следующие основные задачи:

- обоснование необходимости применения и разработка модели построения дополнительных граничных условий, получаемых из основного дифференциального уравнения краевой задачи с использованием исходных (классических) граничных условий;

- применение дополнительных граничных условий с целью определения собственных чисел краевой задачи Штурма-Лиувилля при моделировании нестационарной задачи теплопроводности путем совместного использования метода разделения переменных и ортогонального метода Бубнова-Галеркина;

- разработка математической модели получения аналитических решений краевых задач теплопроводности и тепломассопереноса на основе введения фронта температурного возмущения и дополнительных граничных условий;

- разработка методов построения изотерм и определение скоростей их перемещения по пространственной координате во времени на основе полученных аналитических решений с использованием дополнительных граничных условий;

- разработка алгоритмов и комплекса программ, реализующих предложенные в диссертации аналитические методы решения;

- разработка математической модели решения обратных задач теплопроводности с целью определения граничных условий теплообмена (коэффициентов теплоотдачи) на основе использования полученных в диссертации аналитических решений прямых задач.

12.11.2009. E. A. Андреева (аспирант) каф. прикладной математики и информатики Самарского государственного технического университета

«Структурно-феноменологическая модель реологического деформирования разупрочняющихся и нелинейно-упругих материалов и её приложения»

(доклад по материалам диссертации на соискание учёной степени кандидата наук по специальности 01.02.04)

Обсуждаются проблемы построения математических моделей, по разному детализирующих материал, и позволяющих перейти от микро- к макросвойствам. Показано, что для более адекватного отражения процессов неупругого деформирования, наряду с феноменологическими макротеориями, параллельно развиваются теории, базирующиеся на учете микронеоднородности развития необратимых деформаций и основанные на структурных математических моделях, в основе которых лежит моделирование неоднородностей материала конструкционной неоднородностью. Это, с одной стороны, даёт представление о том, каким образом формируются макроскопические характеристики материала и позволяет более обоснованно выбрать подходящий вариант феноменологической теории в виде интегрального или дифферен- 
циального оператора, связывающего тензоры деформаций и напряжений, внутренние структурные параметры и время, а также, оптимальным образом спланировать определяющий эксперимент для идентификации параметров оператора. С другой стороны, структурные модели с минимально возможным числом параметров можно использовать вместо физических уравнений при решении краевых задач для сред со сложными реологическими свойствами, для которых затруднительно построить феноменологическую модель.

Разработана структурная модель стержневого типа, элементы которой наделены простейшими деформационными свойствами: упругостью, идеальной пластичностью и нелинейной вязкостью, на основе которой решены следующие задачи:

- исследовано неупругое реологическое деформирование и разрушение разупрочняющихся металлических материалов на закритической стадии в условиях сложного и одноосного напряженных состояний;

- исследован эффект Баушингера на стадии разупрочнения и показано, что наблюдается его инверсия по сравнению со стадией упрочнения;

- исследованы неклассические эффекты в процессах деформирования при постоянной скорости нагружения в условиях ползучести, показано, что предел текучести материала зависит от скорости деформации в процессе нагружения;

- разработан метод решения одномерных краевых задач на основании структурной модели на стадии разупрочнения материала в условиях пластичности и ползучести;

- описан и исследован эффект влияния деформации ползучести на нелинейноупругую деформацию микронеоднородных материалов в условиях одноосного и сложного напряженного состояний.

Приводятся результаты численных расчетов, иллюстрирующих рассматриваемые механические эффекты. Выполнена экспериментальная проверка полученных теоретических результатов.

19.11.2009. Л. В. Коваленко (аспирант) каф. прикладной математики и информатики Самарского государственного технического университета

«Исследование краевых эффектов стохастически неоднородных элементов конструкций при установившейся ползучести» (доклад по материалам диссертации на соискание учёной степени кандидата наук по специальности 01.02.04)

Научно-технический прогресс, достижения в сфере высоких технологий диктуют необходимость в глубоком и детальном изучении свойств используемых материалов. При эксплуатации материалов на производстве необходимо учитывать, что все они обладают той или иной степенью неоднородности. Эта неоднородность в материалах может возникать из-за различных технологических и структурных факторов, случайных изменений в параметрах технологических процессов при изготовлении изделия. Структурная неоднородность существенно влияет на процессы деформирования твердых тел, что приводит к их преждевременному разрушению.

Как правило задачи на ползучесть решаются в детерминированной постановке. Однако, если принимать во внимание естественный разброс опытных данных, то становится ясно, что этот подход оказывается явно недостаточным, т.к. он не может объяснить ряд механических эффектов, возникающих в результате неоднородности материала. Одним из таких эффектов является краевой эффект. Суть его состоит в том, что вблизи границ тела со структурной неоднородностью имеется пограничный слой, в котором возникает концентрация напряжений, причем она может достигать достаточно большой величины. Поэтому их неучет в расчетах на ползучесть может привести к нежелательным последствиям - к переоценке прочностных свойств материалов. 
В связи с этим постановки задач предлагается принимать в стохастической форме. Случайность вводится в определяющие соотношения ползучести, взятые в соответствии с нелинейной теорией вязкого течения, с помощью случайной функции с известными характеристиками (математическое ожидание и дисперсия), которая описывает возмущения реологических свойств материала.

Рассматривается приближенное численное решение задачи о растяжении круговой пластины с малым отверстием, аналитическое решение двумерной задачи о растяжении стохастически неоднородной полуплоскости, аналитическое решение пространственной краевой задачи.

Краевые задачи решаются приближенно по методу малого параметра с учетом первого приближения. На основе полученного приближенного решения проводится статистический анализ и исследуются основные особенности краевого эффекта.

Показано, что разброс случайного поля напряжений и деформаций в пограничном слое может быть намного больше, чем в глубине стохастически неоднородной среды. Исследованы такие характеристики как разброс случайного поля напряжений и деформаций, концентрация и коэффициент вариации напряжений на границе среды в зависимости от различных параметров материала и нагружения.

Разработаны методики и алгоритмы расчета на надежность в условиях установившейся ползучести по деформационному критерию отказа и критерию длительной прочности на основе приближенных численных решений стохастической краевой задачи о растяжении круговой пластины с малым отверстием и решен ряд новых модельных задач оценки надежности для некоторых значений параметров материала и внешних нагрузок.

Разработанные методики и алгоритмы позволяют более обоснованно подходить к вопросу о надежности, работоспособности и ресурсу элементов конструкций.

03.12.2009. Д. В. Иванов (аспирант) каф. сопротивления материалов Самарского государственного аэрокосмического университета им. ак. С. П. Королёва

«Прогнозирование предела выносливости упрочнённых деталей с концентраторами напряжений при нормальной температуре и в условиях ползучести» (доклад по материалам диссертации на соискание учёной степени кандидата наук по специальности 01.02.06)

Снижение материальных и трудовых затрат при создании и изготовлении более совершенных машин с одновременным повышением их срока службы, надёжности, снижением массы является основной задачей современного машиностроения, транспорта, авиа- и ракетостроения. Усложнение функциональных возможностей, повышение требований к надёжности, долговечности и материалоёмкости конструкций приводит к появлению всё большего числа деталей сложной формы с резкими концентраторами напряжений. Так как конструктивные методы повышения прочности при переменных нагрузках приводят к увеличению массы конструкций и числа комплектующих, усложнению технологии изготовления, ухудшают унификацию и стандартизацию, основным резервом повышения сопротивления усталости деталей с концентраторами напряжений является применение современных видов и средств поверхностного упрочнения деталей машин.

Методы поверхностного упрочнения основаны на использовании благоприятного эффекта сжимающих остаточных напряжений, влияние которых особенно возрастает в условиях концентрации напряжений. При нормальной температуре остаточные напряжения в поверхностном слое отличаются стабильностью. При работе деталей в области высоких температур их положительное влияние уменьшается вследствие релаксации. В связи с этим тема диссертации, посвящённая прогнозированию предела выносливости упрочнённых деталей с концентраторами напряжений при нормальной температуре и в условиях ползучести, является актуальной.

Обеспечение надёжности и безопасности машин за счёт повышения точности прогнозирования предела выносливости упрочнённых цилиндрических деталей в 
случае изгиба при нормальной температуре и в условиях ползучести на основе исследования остаточных напряжений в образцах и деталях с концентраторами напряжений.

Автором поставлены и решены следующие взаимосвязанные научные задачи:

- проведение теоретического и экспериментального исследований перераспределения остаточных напряжений в цилиндрических деталях, изготовленных с использованием различных методов обработки поверхностным пластическим деформированием (ППД) и опережающим поверхностным пластическим деформированием (ОППД);

- расчётно-экспериментальное определение остаточных напряжений в деталях с концентраторами после ППД и ОППД с последующей термоэкспозицией;

- оценка влияния остаточных напряжений на сопротивление усталости и прогнозирование предела выносливости упрочнённых деталей с концентраторами напряжений.

В работе исследовались цилиндрические сплошные и полые образцы с концентраторами, характерными для деталей машин, после различного вида упрочняющих обработок при нормальной температуре и после термоэкспозиции, при этом применялись расчётно-экспериментальные методы количественной оценки приращения предела выносливости деталей с концентраторами после поверхностного пластического деформирования при нормальной и повышенной температурах.

На основании решения задачи о перераспределении остаточных напряжений на дне надреза полукруглого профиля определены остаточные напряжения после различных методов поверхностного пластического деформирования и проведена оценка их влияния на сопротивление усталости при изгибе упрочнённых деталей с концентраторами напряжений как при нормальной, так и при повышенной температурах. Разработана методика прогнозирования предела выносливости упрочнённых поверхностным пластическим деформированием деталей.

10.12.2009. A. В. Черняев (аспирант) каф. конструкций и проектирования летательных аппаратов Самарского государственного аэрокосмического университета им. ак. С. П. Королёва

«Оптимизация элементов авиационных конструкций из композиционных материалов в дискретной постановке» (доклад по материалам диссертации на соискание учёной степени кандидата наук по специальности 05.07.02)

Использование новых материалов в конструкциях летательных аппаратов (ЛА) традиционно является одним из основных направлений повышения их весовой отдачи и транспортной эффективности. Для авиационных конструкций перспективным представляется использование волокнистых композиционных материалов (стеклопластики, углепластики и т. д.). Их первоначальное внедрение шло по пути использования в несиловых и легко сменяемых агрегатах - обтекателях, створках, рулях и т. п. Накопленный к настоящему времени опыт эксплуатации таких конструкций показал их высокие функциональные качества. Поэтому сейчас в практике ведущих авиастроительных организаций мира наблюдается переход к существенному использованию высокопрочных слоистых композиционных материалов (KM) в наиболее ответственных силовых агрегатах планера, таких как крыло, фюзеляж, оперение. Весовая (массовая) доля КМ в конструкциях современных гражданских и боевых самолетов доходит до $30 \%$.

В отличие от конструкций из традиционных материалов, при проектировании которых определению подлежат, как правило, только размерные параметры (например, толщины поперечных сечений), в ходе разработки композитных конструкций требуется установление дополнительных сведений, характеризующих структуру 
материала (последовательность чередования слоев, углы ориентации волокон). Существующие подходы, основанные на упрощенном моделировании поведения КМ (например, создание конструкций квазиизотропной структуры), часто не позволяют реализовать в многослойных композициях потенциально высокие свойства однонаправленных КМ. Поэтому их эффективное использование в конструкциях ЛА требует применения методов оптимального проектирования.

Специфика композитной конструкции как объекта оптимизации состоит в следующем. Во-первых, технологические особенности строения многослойных композитов, составленных, как правило, из монослоев фиксированной толщины, определяют дискретную природу ряда задач оптимизации их параметров и структуры. Однако в большом числе работ эти задачи ставятся и решаются в непрерывной постановке. Во-вторых, многомерность пространства проектных параметров усложняет поиск глобального экстремума целевого функционала. Успешное решение такой задачи требует применения адекватного метода оптимизации. Данная характеристика позволяет рассматривать в этом качестве так называемые генетические алгоритмы, относящиеся к новому направлению в оптимизации - эволюционным вычислениям.

В соответствии с этим, в работе разрабатываются методика, алгоритмы и программное обеспечение для проектирования элементов авиационных конструкций из волокнистых композиционных материалов с учетом широкого спектра ограничений по прочности, жесткости и технологических особенностей их строения.

На основе разработанного программного обеспечения решена практическая задача проектирования композитного крыла легкого многоцелевого самолета в двух постановках: квазиизотропной и с оптимизацией параметров внутренней структуры, которая выявила резервы массы силовых элементов от $9 \%$ до $39 \%$ в разных проектах.

Семинар проводится регулярно каждый четверг в 17.00 на кафедре прикладной математики и информатики СамГТУ по адресу: ауд. 501, корп. 8, д. 244, ул. Молодогвардейская, Самара, 443100.

По вопросам выступления на научном семинаре с докладом необходимо связаться с секретарём семинара M. Н. Саушкиным (msaushkin@gmail.com) или с руководителем В.П. Радченко (radch@samgtu.ru). 